\title{
Cluster Formation with Chemical and Energy Feedback
}

\author{
L. Portinari, J. Sommer-Larsen, A.D. Romeo \\ Theoretical Astrophysics Center, Juliane Maries Vej 30, Copenhagen, \\ Denmark
}

\begin{abstract}
We present fully cosmological, hydrodynamical simulations of clusters of galaxies, including star formation, supernova feedback, chemical enrichment and metal-dependent cooling. We investigate the relation between feedback, metal enrichment of the intra-cluster medium (ICM) and the distribution of baryons in the various phases (cold gas+stars and hot ICM) at varying Initial Mass Function and wind efficiency.
\end{abstract}

Our clusters are identified from large scale $\Lambda$ CDM cosmological simulations $\left(\Omega_{M}=0.3, \Omega_{\Lambda}=0.7, \mathrm{H}_{0}=70 \mathrm{~km} \mathrm{sec}^{-1} \mathrm{Mpc}^{-1}, \sigma_{8}=0.9\right)$ and then resimulated with an $\mathrm{N}$-body + SPH code (Sommer-Larsen et al. 2003) at 64 times higher mass resolution, assuming a baryonic fraction $f_{b}=0.12\left(\mathrm{~m}_{D M}=1.9 \times 10^{9} h^{-1} \mathrm{M}_{\odot}\right.$, $\left.\mathrm{m}_{b}=2.6 \times 10^{8} h^{-1} \mathrm{M}_{\odot}\right)$.

The hydrodynamical evolution is followed with SPH by integrating the entropy equation, rather than the energy equation, to avoid spurious loss of entropy in strongly cooling regions and reduce the predicted overcooling and cold fraction (Springel \& Hernquist 2002; Tornatore et al. 2003). Detailed chemical evolution of many elements, including SN Ia products, is computed along with star formation by means of the statistical algorithm by Lia et al. (2002). Metal-dependent radiative cooling follows self-consistently the chemical enrichment of the gas phase. Energy feedback from massive stars is implemented by temporarily halting radiative dissipation of energy in the regions surrounding a starburst; this mimicks the effects of (subgrid) supernova-driven bubble expansion, with a very effective energy coupling between massive stars and the gas phase in the simulations. Such feedback at early epochs is crucial to solve the angular momentum problem and form realistic galaxies (Sommer-Larsen et al. 2003).

Here we briefly report on our simulations of a Virgo-sized cluster $(\mathrm{T} \sim 3 \mathrm{keV}$, $R_{v i r}=1.7 \mathrm{Mpc}$ ). We adopt a Salpeter IMF and keep starburst and feedback efficiency at low levels (Weak Winds); we obtain a final cold fraction (fraction of baryons in stars and cold gas) of $20 \%$ within the virial radius (Fig. 1a, solid line), higher than the usual estimates of $10 \%$ (White et al. 1993; Balogh et al. 2001). If we instead maintain strong starburst and feedback activity throughout the evolution (SuperWinds), the cold fraction is only decreased from $20 \%$ to $15 \%$ (Fig. 1a, dashed line). SuperWinds have in fact two opposing effects: (1) they inject energy into the ICM, but they also (2) inject metals, increasing the cooling efficiency of the ICM. Even assuming the top-heavy Arimoto \& Yoshii IMF (AY, dot-dashed line) and SuperWinds hardly changes the resulting cold fraction: this IMF enriches the ICM to higher levels (Fig. 1cd), inducing a higher cooling and star formation rate (Fig. 1b), which compensates for the much lower 

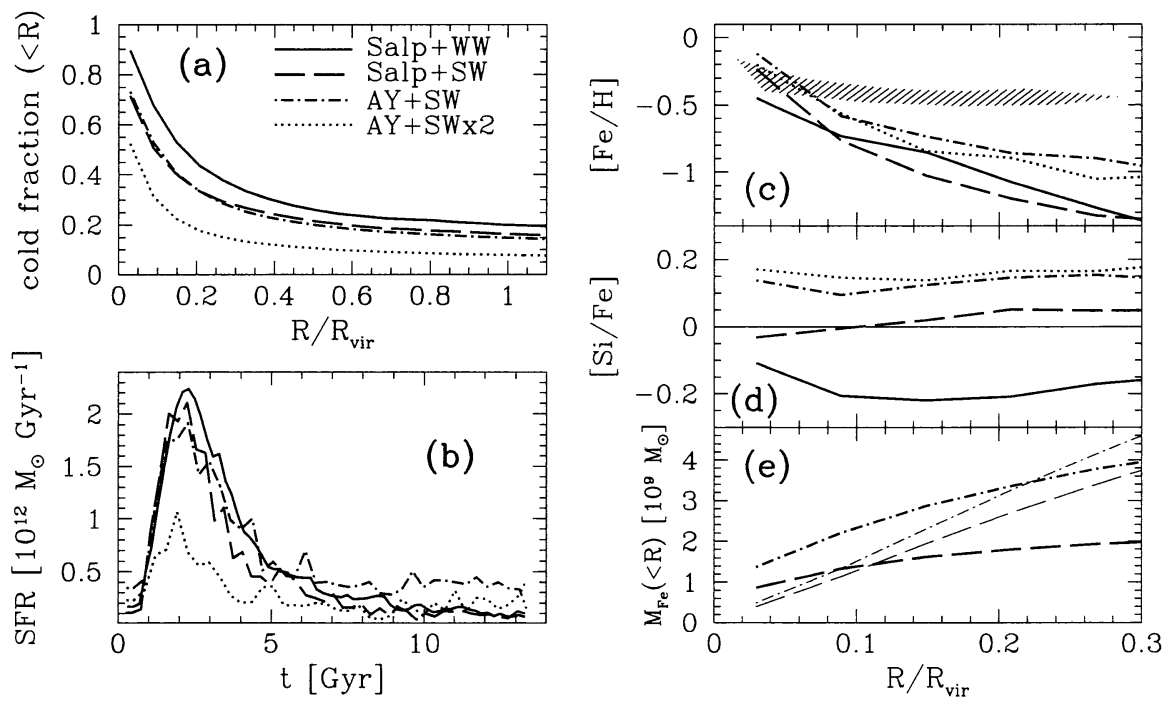

Figure 1. (a) Cold fraction within $R$. (b) Star Formation Rate. (c) Iron abundance profiles vs. the observed one (De Grandi \& Molendi 2001; shaded region). (d) $[\mathrm{Si} / \mathrm{Fe}]$ ratio. (e) Cumulative iron mass in the ICM, compared to that expected for an average abundance of 0.3 solar (thin lines).

"locked-up fraction" in long-lived stars and remnants $(50 \%$ for AY, $70 \%$ for Salpeter). Only by artificially doubling the feedback efficiency - without a corresponding increase in metal production - cooling and star formation are significantly damped, and the cold fraction falls to $10 \%$ (Fig. 1ab, dotted line). We note however that the observational estimates of the cold fraction rely on the assumed stellar mass-to-light ratio (M/L); for a M/L consistent with a Salpeter or AY IMF and the old ages typical of the bulk of the stellar population in clusters, cold fractions of 15-20\% are not excluded (Moretti et al. 2003; Portinari 2003).

SuperWinds significantly increase the metallicity of the ICM; SN II products in particular are more efficiently shed by the starbursts, increasing the $[\alpha / \mathrm{Fe}]$ ratio (Fig. 1cd). The trend is further enhanced with the top-heavy AY IMF. Our iron abundance gradients are much steeper than the observed one (Fig. 1c), a standard problem in simulations. It is then more instructive to compare the cumulative iron mass in the ICM to that expected for an average abundance of 0.3 solar (Fig. 1e, thick vs. thin lines). The Salpeter IMF falls short by a factor of two, while the AY IMF produces about the necessary iron mass out to the observed radius (see also Valdarnini 2002) — but could hardly keep the trend at larger radii. 


\section{References}

Balogh, M.L., Pearce, F.R., Bower, R.G., \& Kay, S.T. 2001, MNRAS, 326, 1228

De Grandi, S., \& Molendi, S. 2001, ApJ, 551, 153

Lia, C., Portinar,i L., \& Carraro, G. 2002, MNRAS, 330, 821

Moretti, A., Portinari, L., \& Chiosi, C. 2003, A\&A, 408, 431

Portinari, L., Moretti, A., Chiosi, C., \& Sommer-Larsen, J. 2003, ApJ submitted Sommer-Larsen, J., Götz, M., \& Portinari, L., 2003, ApJ, 596, 47

Springel, V., \& Hernquist, L. 2002, MNRAS, 333, 649

Tornatore, L., et al. 2003, MNRAS, 342, 1025

Valdarnini, R. 2002, MNRAS, 339, 1117

White, S.D.M., Navarro, J.F., Evrard, A.E., Frenk, C.S., 1993, Nature, 366, 429 